Bundesgesundheitsbl 2019 $\cdot 62: 289-295$ https://doi.org/10.1007/s00103-019-02907-z Online publiziert: 13. Februar 2019

(c) Der/die Autor(en) 2019

CrossMark

\author{
Katja Dierich ${ }^{1} \cdot$ Maria-Theresia Nicolai $^{2} \cdot$ Henrieke Franzen $^{2}$ \\ ' Qualitätsverbund Netzwerk im Alter - Pankow e. V. (QVNIA e.V.), Berlin, Deutschland \\ ${ }^{2}$ Zentrum für Bewegungsförderung Berlin, Gesundheit Berlin-Brandenburg e. V., Berlin-Brandenburg, \\ Deutschland
}

\title{
Umsetzung des Lübecker Modells Bewegungswelten in der Pflege. Kooperation auf kommunaler Ebene zur Stärkung von Prävention und Gesundheits- förderung in Berlin-Pankow
}

Präventionspotenzial auf (siehe Beitrag von Kruse et al. in diesem Heft).

Auf der Landesebene zielt zudem die für Gesundheit zuständige Senatsverwaltung darauf $\mathrm{ab}$, der älter werdenden Bevölkerung und den damit einhergehenden Herausforderungen mit Strategien zu begegnen, die insbesondere die Stärkung und Weiterentwicklung von Gesundheits- und Versorgungsstrukturen vorsehen. Die operative Umsetzung erfolgt durch lokale Akteure, wie z.B. das bezirkliche Netzwerk Qualitätsverbund Netzwerk im Alter - Pankow e. V. (QVNIA e. V.) mit seiner Mitgliedschaft [3], die mit den integrierten kommunalen Strategien für Gesundheitsförderung sowie den vorhandenen Strukturen und Ressourcen vertraut sind. Vertraut mit den bestehenden Strukturen und vorhandenen Ressourcen gilt es, geplante präventive und gesundheitsförderliche Maßnahmen in den Settings Pflege und Kommune an Netzwerke und Akteure vor Ort anzudocken.

Auf Grundlage der gesetzliche Neuerungen im Setting vollstationäre Pflegeeinrichtungen entwickelte die Bundeszentrale für gesundheitliche Aufklärung $(\mathrm{BZgA})$ in Zusammenarbeit mit der Forschungsgruppe Geriatrie Lübeck 2015 das bundesweite Programm „Lübecker Modell Bewegungswelten“(LMB) zur Bewegungsförderung in stationären
Pflegeeinrichtungen ([4]; siehe Beiträge von Krupp et al. und Ralf et al. in diesem Heft).

Zur Implementierung des LMB in städtische Strukturen einer Metropole, wie der Bundeshauptstadt Berlin, wurde modellhaft das Projekt „Adaptation und Umsetzung des Lübecker Modells Bewegungswelten in der Modellregion Pankow - Niedrigschwellige Bewegungsangebote in Pflegeeinrichtungen für ältere Menschen" in der Bezirksregion Berlin-Pankow initiiert. Im Mittelpunkt des Projektes steht der Transfer des Bundesprogrammes LMB unter Berücksichtigung lokaler Strukturen mithilfe eines etablierten Netzwerkes. Sowohl die nachhaltige Umsetzung von Bewegungsförderung in der Pflege als auch die Sensibilisierung für Prävention und Gesundheitsförderung im Setting ist hierbei zentral. Der vorliegende Beitrag stellt das Modellprojekt vor und untersucht, welche Voraussetzungen für eine erfolgreiche Umsetzung gegeben sein müssen. 


\section{Prävention und Gesundheits- förderung in der Pflege durch ein regionales Netzwerk}

\section{Hintergrund und \\ Ausgangssituation}

Um auf die wachsende Zahl hochaltriger Menschen zu reagieren, formulierte die Senatsverwaltung für Gesundheit, Pflege und Gleichstellung (SenGPG) im Jahr 2016 eine Rahmenstrategie $80^{\text {plus }}$ zur Verbesserung der gesundheitlichen und pflegerischen Versorgung hochaltriger Menschen [5]. Gleichzeitig konnte das Berliner Gesundheitsziel „Selbstständigkeit und Lebensqualität im Alter erhalten" der Landesgesundheitskonferenz Berlin um die Zielgruppe der älteren Menschen, die in stationären Einrichtungen leben, erweitert werden [6].

Die Berliner Gesundheitsziele sowie die integrierten kommunalen Strategien für Gesundheitsförderung (Präventionsketten) bilden den Orientierungsrahmen für das Aktionsprogramm Gesundheit (APG) der SenGPG $[7,8]$. Durch das Aktionsprogramm sollen im Land Berlin Prävention und Gesundheitsförderung gestärkt und insbesondere Bürger in schwieriger sozialer Lage einen besseren Zugang zu präventiven und gesundheitsförderlichen Maßnahmen haben. Bei der derzeitigen Entwicklung einer Berliner Strategie für Bewegungsförderung geht es darum, ressort- und ebenenübergreifend Kooperationen auf- und auszubauen und Ressourcen zu bündeln, um die Lebenswelten der Berliner bewegungsförderlicher zu gestalten und Menschen dabei Lust auf Bewegung zu machen. Ein wichtiges Ziel ist die Verbesserung der gesundheitlichen Chancengleichheit.

Die Umsetzung des Modellprojekts „Lübecker Modell Bewegungswelten“ (LMB) ist schließlich ein Ergebnis der Fachveranstaltung APG-Dialogplattform „Strukturen kommen in Bewegung", auf der das Modellprojekt als Teil des Bundesprogramms „Älter werden in Balance" der BZgA vorgestellt und mit Berliner Akteuren diskutiert wurde [9]. Seit Mitte Juni 2017 wird das LMB als Konzept zur präventiven Bewegungsförderung für Menschen mit pflegerischem Unterstützungsbedarf durch den QVNIA e. V. in der Modellregion Berlin-Pankow erprobt und erfolgreich umgesetzt.

Aufgrund der demografischen Struktur und der bereits vorhandenen Vernetzung lokaler Partner durch den QVNIA e. V. eignete sich diese Bezirksregion besonders für die Umsetzung des LMB. Zudem ordnet sich das Modellprojekt in die bereits vorhandenen integrierten kommunalen Strategien für Gesundheitsförderung „Gesund leben in Pankow - in jedem Alter" ein.

Im Bezirk Pankow lebten zum Stand vom 31.12.2017 insgesamt 402.289 Menschen. Das Durchschnittsalter lag bei 40,7 Jahren. Insgesamt waren 14,9\% (34.923 Menschen) der Bevölkerung 65 Jahre und älter.

Zum Stand 2017 wurden 2835 Pflegebedürftige durch ambulante Pflegedienste in ihrer Häuslichkeit bzw. im altersgerechten Wohnen versorgt. Insgesamt 3368 Pflegebedürftige lebten in vollstationären Einrichtungen. Pankow verfügt aktuell über 32 vollstationäre Einrichtungen, elf Tagespflegen (zumeist spezialisiert auf Menschen mit Demenz) sowie sechs spezifische Anlagen des altersgerechten Wohnens.

Zum Start des Projektes wurde durch den QVNIA e.V. festgestellt, dass nur in vereinzelten Einrichtungen ein Programm zur präventiven Bewegungsförderung für Menschen mit pflegerischem Unterstützungsbedarf umgesetzt wurde. Vorgehalten wurden in allen Einrichtungen unterschiedliche Angebote der Betreuung, die auch Bewegungsangebote beinhalteten (wie z. B. Sitztanz, Rollatorentanz, Gymnastikgruppen).

\section{Regionale Struktur}

Seit Mitte Juni 2017 wird das LMB als Konzept zur präventiven Bewegungsförderung für Menschen mit pflegerischem Unterstützungsbedarfdurch den QVNIA e. V. in der Modellregion Berlin-Pankow erprobt und erfolgreich umgesetzt.

Der QVNIA e.V. ist ein gemeinnütziger Verein und engagiert sich seit dem Jahr 2000 für eine bessere gesundheitliche und soziale Versorgung in BerlinPankow, um Strukturen in der Altenhilfe nachhaltig und kontinuierlich zu verbessern. Der QVNIA e. V. arbeitet aktuell mit
64 Mitgliedseinrichtungen und weiteren 40 regionalen sowie überregionalen Partnern der Gesundheits- und Sozialversorgung (z.B. Krankenhäusern, Therapeuten, voll- und teilstationären sowie ambulanten Pflegeeinrichtungen) zusammen, um eine wohnortnahe und koordinierte Versorgung von akut oder chronisch kranken sowie pflege-, hilfs- und/oder rehabilitationsbedürftigen Menschen zu gewährleisten.

Der Verein hat im Rahmen seiner Vernetzung sukzessive ein Qualitätsmanagement aufgebaut und arbeitet an der schnittstellenübergreifenden Qualitätssicherung. $\mathrm{Zu}$ den zentralen $\mathrm{Ar}$ beitsweisen des QVNIA e.V. zählen u. a. die Umsetzung von Projekten, Gremienarbeit, Öffentlichkeitsarbeit zur Aufklärung der Bevölkerung sowie die vernetzte Fortbildung. Über vereinsinterne Richtlinien wird eine qualitativ hochwertige Vernetzung der einzelnen Mitglieder erreicht und sichergestellt. Die Richtlinien werden in den Qualitätszirkeln des Netzwerkes erarbeitet und durch die Mitgliederversammlung verabschiedet.

Des Weiteren arbeitet der QVNIA e. V. in regionalen und berlinweiten Arbeitsgremien zur qualitativen vernetzten Versorgung von älteren Menschen und ihren Angehörigen aktiv mit. Neben der Verbesserung der regionalen Struktur-, Prozess- und Ergebnisqualität der Versorgung fördert der Qualitätsverbund die Transparenz für die Bürger, speziell für Betroffene und deren Angehörige in Pankow. Nähere Informationen sind unter www.qvnia.de zu finden.

Insgesamt stellt der QVNIA e. V. ein nachhaltiges regionales Netzwerk zur gesundheitlichen und pflegerischen Versorgung im Großbezirk Pankow dar, das eng mit dem Bezirksamt Pankow sowie mit der SenGPG zusammenarbeitet. Zu den weiteren zentralen Zielen des Verbundes zählen, Prozesse der Prävention und Gesundheitsförderung im Alter gemeinsam mit den Partnern zu entwickeln und präventive Maßnahmen kommunal mit den Partnereinrichtungen umzusetzen [3]. 
Bundesgesundheitsbl 2019·62:289-295 https://doi.org/10.1007/s00103-019-02907-z

(c) Der/die Autor(en) 2019

K. Dierich · M.-T. Nicolai · H. Franzen

\section{Umsetzung des Lübecker Modells Bewegungswelten in der Pflege. Kooperation auf kommunaler Ebene zur Stärkung von Prävention und Gesundheitsförderung in Berlin-Pankow}

\section{Zusammenfassung}

Im Zuge des Präventionsgesetzes und des damit verbundenen neuen Präventionsauftrags der Pflegekassen finden seit 2016 vermehrt präventive und gesundheitsfördernde Aktivitäten in stationären Pflegeeinrichtungen statt. Das „Lübecker Modell Bewegungswelten“ (LMB) als Element des Programms „Älter werden in Balance" der Bundeszentrale für gesundheitliche Aufklärung (BZgA) wurde als körperlich, geistig und sozial aktivierendes Präventionsprogramm für ältere Menschen mit körperlichen und kognitiven Einschränkungen entwickelt.

Der regionalen Umsetzung des LMB in stationären Pflegeeinrichtungen, im altersgerechten Wohnen und in der Tagespflege dient das Berliner Projekt „Adaption und Umsetzung des Lübecker Modells Bewegungswelten in der Modellregion Pankow - Niedrigschwellige Bewegungsangebote in Pflegeeinrichtungen für ältere Menschen“. Dieses Modellprojekt des regionalen Qualitätsverbunds Netzwerk im Alter - Pankow e. V. (QVNIA e. V.) wird im vorliegenden Beitrag vorgestellt. Beleuchtet werden die für die Umsetzung relevanten lokalen Strukturen und Kooperationen sowie die daraus auch programmbedingt resultierenden Anforderungen an die beteiligten Akteure. Mit dem Ziel der nachhaltigen Umsetzung von Bewegungsförderung im Setting Pflege werden ein möglicher Implementierungspfad und qualitätssichernde Maßnahmen vorgestellt.

Der Praxistransfer hat gezeigt, dass sich das Konzept des LMB auch in großstädtischen Strukturen anwenden lässt. Bei der Umsetzung müssen regionale Rahmenbedingungen berücksichtigt werden. Notwendig ist eine zentrale Steuerung vor Ort mit der entsprechenden strukturellen und prozessualen Expertise sowie qualitätssicherndem Vermögen. Während der Modellphase konnten positive Erfahrungen gewonnen werden, aus denen Handlungsempfehlungen zur Fortführung und Weiterentwicklung abgeleitet werden.

Schlüsselwörter

Prävention · Bewegungsförderung · Stationäre Pflegeeinrichtungen · Pflegebedürftige · Netzwerkstrukturen

\section{Implementation of the Lübeck model of themed movement activities in nursing. Cooperation at the local level to strengthen the prevention and health promotion in Berlin-Pankow}

\section{Abstract}

In accordance with Prevention Act law and the associated new prevention mandate of the nursing care funds, more preventive and health promoting activities have been taking place in inpatient care facilities since 2016. The Lübeck model of themed movement activities as a part of the program "Grow older in Balance" of the Federal Center for Health Education was developed as a physical, mental, and socially activating prevention program for elderly people with physical and cognitive impairments.

The regional implementation of the Lübeck model in inpatient care facilities, facilities for age-appropriate living, as well as in daycare, serves the Berlin project "Adaptation and implementation of the Lübeck model of themed movement activities in the model region of Pankow - Low threshold exercise offers in nursing facilities for the elderly." This model project of the regional network Qualitätsverbund Netzwerk im Alter - Pankow e.V. (QVNIA e. V.) is presented in the current article.

The relevant local structures and cooperation as well as the resulting and program-related requirements for participating actors are highlighted. With the aim of sustainable implementation of physical activity promotion in the nursing setting, a possible implementation path and quality assurance measures are presented.

The transfer has shown that the concept of the Lübeck model can also be implemented in metropolitan structures. However, the implementation requires the consideration of the regional framework conditions. A centralized local control with the corresponding structural and procedural expertise as well as quality assurance assets is necessary. Positive experiences in the implementation of the program were acquired during the model phase and recommendations for the continuation and further development can be derived.

Keywords

Prevention · Movement-promoting · Inpatient care facilities - Care-dependent person . Network

\section{Regionale Implementierung}

\section{Vorhaben}

Zum 15.06.2017 konnte mit dem Transfer und der Implementierung des Modellprojekts „Adaption und Umsetzung des Lübecker Modells Bewegungswelten in der Modellregion Pankow Niedrigschwellige Bewegungsangebote in Pflegeeinrichtungen für ältere Men- schen" begonnen werden. Die Laufzeit des durch die BZgA beauftragten und geförderten Projekts beträgt eineinhalb Jahre (15.07.2017-31.12.2018).

Unter Berücksichtigung bezirklicher Besonderheiten und etablierter Strukturen übernimmt der QVNIA e.V. als Träger die zentrale bezirkliche Koordination. Weitere Partner, die die Umsetzung des kooperativen Modellprojekts steuernd begleiten, sind das Bezirksamt
Pankow mit der Abteilung „Qualitätsentwicklung, Planung und Koordination des öffentlichen Gesundheitsdienstes“ (QPK) und das Zentrum für Bewegungsförderung Berlin (ZfB Berlin) in Trägerschaft von Gesundheit Berlin-Brandenburg e.V., gefördert durch die SenGPG. Das ZfB Berlin ist eine etablierte Koordinierungsstelle für Bewegungsförderung auf Landesebene. Seine Aktivitäten und Maßnahmen sind 
darauf ausgerichtet, niedrigschwellige Bewegung $\mathrm{zu}$ fördern und diese bedarfsgerecht und zielgruppenspezifisch in Lebenswelten nachhaltig zu verankern, um Berlinern in benachteiligten Lebenslagen bewegungsförderliche und bewegungsfreundliche Rahmenbedingungen als Beitrag für ein gutes und gesundes Leben zu bieten [10].

Im Rahmen der Projektlaufzeit wurden sich folgende Ziele gesetzt:

\section{Übergeordnetes Ziel:}

Implementierung eines niedrigschwelligen Bewegungsangebotes in Pflegeeinrichtungen sowie Einrichtungen des altersgerechten Wohnens ${ }^{1}$, um die Gesundheit und Lebensqualität älterer Menschen $\mathrm{zu}$ verbessern und $\mathrm{zu}$ erhalten. Der Erhalt und Ausbau der Selbsthilfefähigkeit, Mobilität, Beweglichkeit, geistigen Leistungsfähigkeit älterer Menschen sowie die Stärkung sozialer Kontakte nehmen hierbei eine zentrale Rolle ein.

Formulierte Unterziele:

- Verbindliche und nachhaltige Netzwerkerweiterung zur Umsetzung, u.a. durch die Qualifizierung von LMB-Übungsleitern, kommunale Strukturentwicklung

- Etablierung des LMB in bis zu 30 vollstationären Pflegeeinrichtungen/ Einrichtungen des altersgerechten Wohnens Pankows

- Qualitätsentwicklung und -sicherung

- Sicherung der Nachhaltigkeit

Zur Erreichung der genannten Ziele übernimmt der QVNIA e.V. in der bezirklichen Projektsteuerung, -umsetzung und -begleitung die zentrale Rolle. Er übernimmt sowohl die Gewinnung als auch die Begleitung der teilnehmenden Einrichtungen und Übungsleitenden. Zur Qualitätssicherung setzt er u. a. partnerübergreifende Qualitätszirkel um sowie weitere qualitätssichernde Instru-

\footnotetext{
1 Aktuell besteht keine allgemeingültige Definition zu Einrichtungen des altersgerechten Wohnens. Im Folgenden wird diese Begrifflichkeit als spezialisiertes Setting in der Kommune verstanden, welches für ältere Menschen mit und ohne professionellen Hilfebedarf ein Wohnangebot bereithält und zusätzliche Angebote der Serviceleistung und der barrierearmen Wohnmöglichkeit umfasst.
}

mente (z. B. Coachings der Übungsleitenden, Auswertung und Controlling der Beteiligung, Befragungen der beteiligten Akteure zur Wirksamkeit auf Teilnehmerebene, Zufriedenheitsgespräche mit den handelnden Akteuren). Neben der regionalen Öffentlichkeitsarbeit zielt die regionale Etablierung des Programms mittels der Netzwerkstrukturen auf die nachhaltige Verankerung des Programmes ab.

Die Übernahme der Aufgabenbereiche erfolgt einerseits durch die Geschäftsführung des QVNIA e.V., andererseits auch durch einen Projektmitarbeiter als sportliche Leitung mit der Qualifikation Sportwissenschaft und LMB-Trainer.

Zur bezirklichen politischen Verankerung des Modellprojektes liegt die Schirmherrschaft bei dem Pankower Bezirksstadtrat für Schule, Sport, Facility Management und Gesundheit und wird durch die dort ansässige Organisationseinheit QPK unterstützt. Das ZfB Berlin unterstützt im Rahmen der Prozessbegleitung die Umsetzung und den Transfer auf Landes- und Bundesebene. Zur Sicherung der Nachhaltigkeit, kontinuierlichen Steuerung sowie Begleitung der Projektumsetzung in Pankow werden mit den Kooperationspartnern (QVNIA e. V., Bezirksamt Pankow, ZfB Berlin) Steuerungsrunden durchgeführt.

Die formative Evaluation des Projektes wurde von der Universität Bielefeld übernommen.

\section{Umsetzung und Zwischenergebnis}

Aktuell setzen insgesamt 21 vollstationäre Einrichtungen, eine Tagespflege sowie zwei altersgerechte Wohnanlagen das LMB unter Begleitung des QVNIA e.V. um. Bis auf eine haben alle Einrichtungen ihren Standort in Pankow. Somit konnten $80 \%$ aller potenziellen Settings für das Programm durch den QVNIA e.V. in Pankow erreicht werden. Die Anleitung der Bewegungsgruppen erfolgt aktuell mithilfe von über 30 qualifizierten LMB-Übungsleitern.

\section{Regionaler Implementierungspfad} Die Gewinnung der Partner erfolgte mehrschrittig. Im ersten Schritt wurden Informationsveranstaltungen umgesetzt und alle potenziellen Einrichtungen zum LMB schriftlich informiert. Anschließend wurde telefonisch Kontakt aufgenommen. Mit interessierten Einrichtungen erfolgte eine Inhouseschulung unter Beteiligung der Einrichtungsleitung, teilweise der Pflegedienstleitung und/oder Qualitätsbeauftragten sowie beispielsweise auch ausgewählter Mitarbeitender aus der Pflege und Betreuung. Die Schulung diente der Erläuterung des kompletten Verfahrens zur Umsetzung des LMB.

Auf Grundlage einer schriftlichen Interessensbekundung wurde u. a. eine Person bestimmt, die die langfristige Projektverantwortung in der jeweiligen Einrichtung übernehmen sollte. Nach einer Einschätzung der Einrichtung durch den QVNIA e.V., wählte dieser eine geeignete Person als LMB-Übungsleiter aus und fragte diese an. Im Anschluss kam es zum Vertragsabschluss zwischen dem QVNIA e. V., der Übungsleitung und der jeweiligen Einrichtung, um die Kooperation verbindlich zu regeln.

Zur Unterstützung der Zusammenstellung der Bewegungsgruppe wurde ein sogenanntes Schnuppertraining entwickelt. Dieses wird in der Einrichtung durchgeführt und hat einen zeitlichen Umfang von einer Stunde. Es dient der gesamten Bewohnerschaft und allen Mitarbeitenden als offenes Angebot zur Information und Möglichkeit, erste praktische Erfahrungen mit dem LMB zu sammeln. Das Schnuppertraining hat sich als besonders förderlich für die Gründung und interne Bekanntmachung einer Bewegungsgruppe erwiesen.

Die interessierten Teilnehmer melden sich im Nachgang schriftlich über die einrichtungsspezifische Projektleitung an, die in Abstimmung mit dem verantwortlichen Arzt steht.

\section{Teilnahme am Bewegungsprogramm}

Unter Erfüllung der Teilnahmevoraussetzungen (mindestens einmal wöchentlich Unterstützungsbedarf in der Pflege, stehfähig ggf. mit Hilfsmittel, kognitiv befähigt und motiviert zur kontinuierlichen Teilnahme) nehmen regelmäßig im Durchschnitt acht bis zwölf Personen zweimal wöchentlich zu je $45 \mathrm{~min}$ 
an den Bewegungsgruppen des LMB teil. Das LMB ist ein vollstandardisiertes Bewegungsprogramm. Insgesamt werden in Berlin-Pankow aktuell 17 unterschiedliche Bewegungswelten durchgeführt. Als Bewegungswelten werden Themen verstanden, wie z. B. „am Strand“ oder „im Wald“, „Apfelernte“ oder „Hausbau“. Eine Bewegungseinheit steht jeweils unter einem vom Übungsleiter ausgewählten Thema. $\mathrm{Zu}$ den Bewegungswelten liegt ein standardisiertes Bewegungsprogramm vor. Zur Ergebnissicherung werden die Beteiligung und Kontinuität der Teilnehmenden über den QVNIA e.V. ausgewertet und in Zwischengesprächen mit den Übungsleitenden und der Einrichtung reflektiert und dokumentiert. Aktuell ist eine hohe Kontinuität in den LMB-Gruppen zu verzeichnen, was mit einer hohen Motivation und Freude der Teilnehmer zu begründen ist. Momentan läuft eine Befragung der Teilnehmenden seitens des QVNIA e. V. im Rahmen der Qualitätssicherung. Mit Ergebnissen ist zum Ende des Jahres 2018 zu rechnen. Austritte aus den LMB-Gruppen erfolgen aktuell nur, wenn der gesundheitliche Allgemeinzustand sich verschlechtert.

Ergänzend zum Bewegungsprogramm erhalten die Teilnehmer zu den umgesetzten Bewegungswelten ein tägliches Bewegungsprogramm („Mein tägliches Bewegungsprogramm“, MTB), welches selbstständig bis $\mathrm{zu}$ fünfmal täglich durchgeführt bzw. durch die Betreuungsassistenz vor Ort angeleitet wird. Die Akzeptanz dieses "Moduls“ ist unterschiedlich. Einige Teilnehmer setzen es mit hoher Motivation um und haben sogar „Lerngruppen“ gegründet. Dies zeigt auch die soziale Komponente des Programms auf. Überwiegend benötigen jedoch die Teilnehmenden außerhalb der Gruppen Anleitung und Motivation durch die Betreuungs- oder Pflegekräfte, was aufgrund mangelnder personeller Ressourcen nicht immer gelingen kann.

Aktuell wird in jeder der 21 Einrichtungen eine Bewegungsgruppe durchgeführt. In einzelnen Einrichtungen besteht das Interesse, zusätzlich eine weitere Gruppe zu eröffnen. Derzeit nutzen um die 240 Teilnehmer das LMB in Berlin-Pankow. Pro Einrichtung sind es durchschnittlich $11 \%$ der Bewohner- bzw. Gästeschaft, die an dem Bewegungsprogramm teilnehmen. Aktuell werden ausgewählte Einrichtungen ergänzend in ihrer Öffentlichkeitsarbeit unterstützt, um auch Interessenten aus dem Quartier an dem Programm partizipieren zu lassen. Aspekte wie Haftungsrecht und auch Mobilität/Transfer zu den Bewegungsgruppen stellen in Teilen eine operative Herausforderung dar.

\section{Einbindung qualifizierter Übungsleitender}

Die LMB-Bewegungsgruppen werden von qualifizierten LMB-Übungsleitern durchgeführt. Pro Einrichtung gibt es eine hauptverantwortliche und eine stellvertretende Übungsleitung. Die Sicherstellung von Vertreterlösungen übernimmt zusätzlich die sportliche Leitung des QVNIA e.V.

Die Akquise der Übungsleitung erfolgte über die schriftliche Ansprache aller Physio- und Ergotherapeuten Pankows, über die Berliner Universitäten im Bereich Sportwissenschaft, Pankower Sportvereine sowie über Übungsleiterportale. Die Voraussetzungen für die Qualifizierung zur LMB-Übungsleitung sind anhand von definierten Grundqualifikationen formuliert (siehe Beitrag von Ralf et al. in diesem Heft).

Bei Interesse am Programm bzw. zum kollegialen Austausch besteht das kostenfreie Angebot der Hospitation in einer Bewegungsgruppe. Das Schulungsprogramm ist vollstandardisiert, jedoch an regionale organisatorische Rahmenbedingungen angepasst. Es wurde anfänglich eng durch das Referenzteam in Lübeck begleitet und wird nun eigenständig durch den QVNIA e. V. für den Berliner Raum durchgeführt. Die Schulung ist aktuell kostenfrei und steht auch bundesweit für Interessierte zur Verfügung. Aufbauend auf der Grundqualifikation erfolgt eine Weiterqualifizierung mit insgesamt 15 Unterrichtseinheiten zum LMB.

Bisher erfolgten in Berlin-Pankow insgesamt sechs Schulungen zur Übungsleitung mit 63 Teilnehmern, von denen 50 für Berlin-Pankow weiterqualifiziert wurden. Somit konnte ein Übungsleiterpool aufgebaut werden. In insgesamt acht Einrichtungen werden die Bewegungs- gruppen von den weiterqualifizierten internen LMB-Übungsleitern (in den Einrichtungen angestellte Ergo- bzw. Physiotherapeuten) durchgeführt, in 15 Einrichtungen durch externe LMBÜbungsleitende. Nach Abschluss der Grundqualifizierung erfolgt nach ein-bis zweimonatiger praktischer Tätigkeit ein Coaching durch die sportliche Leitung im QVNIA e.V. Nach erfolgreicher Umsetzung erfolgt die Lizenzausstellung durch das Referenzzentrum in Lübeck. Die kontinuierliche Begleitung der Übungsleitenden erfolgt regional durch den QVNIA e.V.

\section{Umsetzung des LMB in den teilnehmenden Einrichtungen}

Zum Start der Bewegungsgruppe erhält die Einrichtung ein Set von Materialien, den sogenannten Lübecker Bewegungskoffer. Im Rahmen eines vom QVNIA e. V. begleiteten Kennenlerntreffens werden die Übungsleiter über die Teilnehmenden, die Zusammenarbeit und die örtlichen Gegebenheiten informiert.

In der Regel werden die Teilnehmer von ihrem Betreuungspersonal zu den Gruppen gebracht und später abgeholt, wenn dies nicht selbstständig erfolgen kann. Zudem ist die Beteiligung des Betreuungspersonals eine wichtige Unterstützung für den Übungsleitenden auch bei der Durchführung des täglichen Bewegungsprogramms (MTB). Gestützt wird die Zusammenarbeit zwischen Betreuungskraft und Übungsleitung durch eine Fortbildung seitens des QVNIA e. V. Hier werden Grundlagen und Besonderheiten zur Bewegung bei älteren Menschen mit präventivem Ansatz sowie praktisch die Anleitung der MTB vermittelt. Insbesondere wird auf die korrekte Durchführung geachtet. Die Fortbildung ist für Pflege(fach)kräfte geöffnet, um auch hier die Zusammenarbeit aller Professionen zu befördern, den Stellenwert der Prävention zu vermitteln und eine Integration des Programms in den Pflegeprozess zu erreichen.

\section{Kontinuierliche Qualitätssicherung und Projektsteuerung}

Nach erfolgreichem Start des Programms erfolgen in regelmäßigen Abständen kurze Zufriedenheitsgespräche sowohl mit 
den Akteuren der Einrichtung, in erster Linie mit dem Projektverantwortlichen, als auch mit den Übungsleitern, um die Umsetzung reibungslos durchzuführen und Erkenntnisse zur Weiterentwicklung des Programms zu erhalten. Bei der Rechnungslegung durch die Übungsleitenden werden stichprobenartig die Planungs- und Dokumentationsbögen qualitativ ausgewertet. Die quantitative Auswertung der Beteiligung erfolgt über die anonymisierten Teilnehmerlisten. Auch dies dient sowohl der Qualitätssicherung als auch der Projektsteuerung.

Seit dem Projektstart wurden insgesamt drei Qualitätszirkel (QZ) unter Beteiligung von Wissenschaftlern und Mitarbeitern des Referenzzentrums Lübeck durchgeführt. An den QZ nahmen ebenfalls die Projektleitungen der Einrichtungen sowie die Betreuungskräfte und Übungsleitenden teil. Die Moderation und Ergebnissicherung erfolgte durch den QVNIA e.V. sowie das ZfB Berlin. Zentrales Thema der QZ war die professions- und einrichtungsübergreifende Auswertung des Umsetzungsprozesses sowie des Konzeptes als solches. Im Ergebnis konnten wertvolle Erkenntnisse zur Weiterentwicklung gewonnen werden. Ein QZ diente darüber hinaus zur Lizenzverlängerung der LMB-Übungsleitenden. Es erfolgte eine Nachschulung mit drei Unterrichtseinheiten seitens des Referenzzentrums in Lübeck. Aktuell werden Gespräche zur Sicherung der Nachhaltigkeit und Fortführung des Programms in Pankow sowie zu einer möglichen Ausweitung in Berlin geführt.

\section{Diskussion}

Netzwerkstrukturen und regionale Koordination als Erfolgsfaktoren

Das inhaltliche Konzept des LMB ist aus der Sicht der Praxis ein Bewegungsprogramm, welches ergänzend zum eigentlichen Versorgungsauftrag von Einrichtungen im kommunalen pflegerischen Setting (vollstationäre Pflegeeinrichtungen, Einrichtungen der Tagespflege und des altersgerechten Wohnens) zur Prävention und Gesundheitsförderung im Alter im Handlungsfeld Bewegung beiträgt. Es zeigte sich, dass das Programm auch in großstädtischen Regionen wie Berlin umgesetzt werden kann. Hierzu bedarf es jedoch der Berücksichtigung regionaler Rahmenbedingungen und des Einsatzes einer regionalen Projektbegleitung durch zentrale, qualifizierte und vernetzte Akteure vor Ort. Dies konnte durch den QVNIA e. V. gelingen, der zu einer zügigen und qualitätsgesicherten Umsetzung des Konzeptes maßgeblich beigetragen hat.

Um die gestellten Programmanforderungen des LMB erfüllen zu können, braucht es strukturierte Maßnahmen zur Koordination, Begleitung und ggf. zur regionalen Anpassung. Hierzu wurde ein Implementierungspfad entwickelt, der die regionalen Gegebenheiten berücksichtigt. Auf diese Weise konnte nicht nur die Verbreitung des Programms ermöglicht werden, sondern auch die notwendigen Maßnahmen zur Organisationentwicklung in Richtung Gesundheitsförderung konnten entwickelt werden. Weiterhin ist eine übergeordnete Prozessbegleitung, wie sie durch das ZfB Berlin erfolgt, unabdingbar. Sie fördert die Verschränkung zwischen lokalen bzw. bezirklichen Maßnahmen und gesamtstädtischen Strukturen sowie gesundheitspolitischen Landesstrategien der Gesundheitsförderung. Zudem bringt sie Expertise soziallagenbezogener Gesundheitsförderung in den Umsetzungsprozess ein. Die Beteiligung des Bezirksamtes erreicht die frühzeitige kommunalpolitische Einbindung und berücksichtigt die bezirklichen Umsetzungsstrategien der Präventionskette.

\section{Bedeutung des LMB für Einrich- tungen und Übungsleitende}

Seitens der Einrichtungen, die das LMB umsetzen, wird das Programm als ein attraktives Angebot gegenüber der Bewohner- bzw. Gästeschaft bewertet, da es zur Gesundheitsförderung beiträgt. Effekte in den Bereichen Mobilität, Kognition sowie im Aufbau neuer sozialer Kontakte konnten bei den Teilnehmenden festgestellt werden. Die Teilnahme erfolgt mit einer hohen Motivation und Kontinuität. Das Prinzip der Bewegungswelten fördert den Austausch zu Lebenserfahrungen zwischen Teilnehmenden und Per- sonal sowie zur übrigen Bewohnerschaft, der zur weiteren Aktivierung beiträgt.

Für die Umsetzung ist eine Anpassung von organisatorischen Abläufen erforderlich. Darüber hinaus bedarf es Maßnahmen zur Berücksichtigung des Programms im Pflegeprozess, zur Information aller Mitarbeitenden sowie zur Fortbildung der Betreuungsassistenten. Die übergreifende Koordination seitens des QVNIA e. V. wurde hierzu als notwendig erachtet, um interne Ressourcen neben dem eigentlichen Versorgungsauftrag nicht zu binden. Um einen deutlich höheren Anteil der Bewohnerschaft partizipieren lassen zu können, wird von den teilnehmenden Einrichtungen eine Erweiterung der möglichen Teilnehmerschaft auf Menschen mit Demenz und stärker eingeschränkte Bewohner gewünscht.

Seitens der Übungsleitenden wurden das standardisierte Vorgehen sowie das festgelegte Motto innerhalb einer Bewegungswelt als äußerst hilfreich für die Planung und Umsetzung der Trainingseinheiten bewertet. Das Angebot an unterschiedlichen Bewegungswelten sowie einer entsprechenden Musik ermöglicht die Umsetzung eines abwechslungsreichen Trainings, welches zur Motivation beiträgt. Die Übungsleitenden stellten bereits nach wenigen Trainingseinheiten positive Effekte in der Mobilität und Selbstständigkeit der Teilnehmenden fest. Es besteht der Wunsch, das Angebot an Bewegungswelten auszubauen und an die Lebenswelten von Städtern anzupassen.

Der regelmäßige Austausch im Rahmen des übergreifenden Qualitätszirkels fördert einen fachlichen Austausch zur praktischen Umsetzung sowie die Vernetzung aller beteiligten Akteure. Handlungsempfehlungen zur qualitativen Weiterentwicklung des Programms konnten einvernehmlich erarbeitet werden.

\section{Ausblick}

Insgesamt eignet sich das LMB als Bewegungsprogramm zur Prävention und Gesundheitsförderung in der voll- und teilstationären Pflege sowie dem altersgerechten Wohnen. Durch die Öffnung der 
Einrichtungen ist auch die Teilhabe von ambulant versorgten Personen möglich, wenn kommunale Voraussetzungen zur Beteiligung geschaffen werden. Unter Berücksichtigung der Modellerfahrungen in Berlin-Pankow weist das Programm ein Potenzial auf, in weitere Berliner Bezirke ausgeweitet zu werden. Hierzu bringen der QVNIA e. V. und das ZfB Berlin grundlegende Erfahrungen aus der Modellphase mit. Die entsprechende Expertise sowie die grundlegende berlinweite Vernetzung weisen beide Partner auf.

Die in der Modellphase beteiligten Akteure und Teilnehmenden sind an einer Fortführung des Programms äußerst interessiert und möchten das Angebot zur Prävention und Gesundheitsförderung nutzen. Dabei müssen die Anforderungen zur Implementierung sich stets an den Rahmenbedingungen vor Ort orientieren, entsprechend angemessen sein und sich ggf. qualitätsgesichert flexibel anpassen lassen.

Die Anpassung der Teilnahmevoraussetzungen, um auch die Zielgruppe der Menschen mit Demenz und andere stärker eingeschränkte Menschen zu erreichen, ist dringend zu empfehlen, um der Bewohnerschaft in Pflegeeinrichtungen gerecht werden zu können. Die Weiterqualifizierung von grundlegend gut ausgebildeten Betreuungsassistenten mit besonderem Kompetenzprofil kann die Implementierung in Einrichtungen befördern und den notwendigen Pool an qualifizierten Übungsleitenden erweitern.

Um die Umsetzung von Maßnahmen zur Prävention und Gesundheitsförderung neben dem eigentlichen Versorgungsauftrag zu ermöglichen, benötigen die beteiligten Einrichtungen zusätzliche Ressourcen. Dies ist für die gewünschte Umsetzung des Gesundheitsförderungsprozesses gemäß dem geltenden Präventionsgesetz zu berücksichtigen.

Zur regionalen Umsetzung des LMBProgramms bedarf es der zentralen Steuerung vor Ort. Der entwickelte Implementierungspfad eignet sich zur qualitätsgesicherten Umsetzung des Programms. Die beschriebenen Maßnahmen zur Koordination, Begleitung und Qualitätssicherung sind zur qualitativen Umsetzung des Programms erforderlich und sollten zentral umgesetzt werden.
Die einzelnen Maßnahmen zur Implementierung müssen zudem weiterführend die Organisationentwicklung in Richtung Prävention und die nachhaltige Umsetzung in den Einrichtungen berücksichtigen.

\section{Korrespondenzadresse}

\section{Katja Dierich}

Qualitätsverbund Netzwerk im Alter - Pankow e.V. (QVNIA e.V.)

Schönhauser Allee 59b, 10437 Berlin,

Deutschland

kontakt@qvnia.de

\section{Einhaltung ethischer Richtlinien}

Interessenkonflikt. K. Dierich, M.-T. Nicolai und $H$. Franzen geben an, dass kein Interessenkonflikt besteht.

Dieser Beitrag beinhaltet keine von den Autoren durchgeführten Studien an Menschen oder Tieren.

Open Access. Dieser Artikel wird unter der Creative Commons Namensnennung 4.0 International Lizenz (http://creativecommons.org/licenses/by/4.0/deed. de) veröffentlicht, welche die Nutzung, Vervielfältigung, Bearbeitung, Verbreitung und Wiedergabe in jeglichem Medium und Format erlaubt, sofern Sie den/die ursprünglichen Autor(en) und die Quelle ordnungsgemäßnennen, einen Linkzur Creative Commons Lizenz beifügen und angeben, ob Änderungen vorgenommen wurden.

\section{Literatur}

1. GKV-Spitzenverband (2018) Leitfaden Prävention in stationären Pflegeeinrichtungen nach $§ 5$ SGB XI. https://www.gkv-spitzenverband.de/media/ dokumente/presse/publikationen/Leitfaden_ Pravention_stationar_2018_barrierefrei.pdf. Zugegriffen:20. Aug. 2018

2. GKV-Spitzenverband (2017) Expertenstandards nach $\S 113$ a SGB XI. https://www. gkv-spitzenverband.de/pflegeversicherung/ qualitaet_in_der_pflege/expertenstandards/ expertenstandards.jsp.Zugegriffen:24. Apr. 2017

3. QVNIA e.V. (2017) Wir über uns. http://www.qvnia. de/wir-uber-uns.Zugegriffen:24. Apr. 2017

4. BZgA - Bundeszentrale für gesundheitliche Aufklärung (2016) Lübecker Modell Lebenswelten. http://www.aelter-werden-in-balance.de/ programme/luebecker-modell/. Zugegriffen: 24 . Apr. 2017

5. Senatsverwaltung für Gesundheit und Soziales Berlin (2016a) 80plus-Rahmenstrategie zur gesundheitlichen und pflegerischen Versorgung hochaltriger Menschen in Berlin Ziele, Maßnahmen und Kooperationspartner. http://www.gesundheitbb.de/fileadmin/ user_upload/MAIN-dateien/GesBB/Materialien/ Projektmaterial/Berlin/80plus_rahmenstrategie. pdf.Zugegriffen:24.Apr. 2017
6. Fachstelle für Prävention und Gesundheitsförderung im Land Berlin (2017) Gesund altern in Berlin.http://www.berlin.gesundheitfoerdern.de/ Gesund-altern-in-Berlin.1415.0.html. Zugegriffen: 24. Apr. 2017

7. Senatsverwaltung für Gesundheit, Pflege und Gleichstellung (2017) Aktionsprogramm Gesundheit. https://www.berlin.de/sen/ gesundheit/themen/gesundheitsfoerderungund-praevention/aktionsprogramm-gesundheit/. Zugegriffen: 24. Apr. 2017

8. Senatsverwaltung für Gesundheit und Soziales (2016b) Pflege- und pflegeunterstützende Angebote in Berlin. Landespflegeplan 2016. https:// www.berlin.de/sen/pflege/_assets/service/ publikationen/2016-06-01_landespflegeplan v02.pdf.Zugegriffen:24. Apr. 2017

9. Koordinierungsstelle Gesundheitliche Chancengleichheit Berlin (2016) Strukturen kommen in Bewegung. Gute Ansätze für Bewegungsförderung in allen Lebensphasen. http://www. gesundheitbb.de/fileadmin/user_upload/MAINdateien/GesBB/Materialien/Projektmaterial/ Berlin/Dialogplattform_APG.pdf.Zugegriffen: 24 . Apr. 2017

10. Zentrum für Bewegungsförderung (2018) Zentrum für Bewegungsförderung. https://www. gesundheitbb.de/Zentrum-fuer-Bewegungsfoer derung.1362.0.html?\&L=0Der. Zugegriffen: 1 . Aug. 2018 